\title{
Signal-to-Noise Ratio as a Function of Imaging Parameters
}

\section{OVERVIEW}

The degree to which noise affects a measurement is generally characterized by the signal-to-noise ratio (SNR, as measured by the ratio of the voxel signal to the noise standard deviation). SNR is the key parameter for determining the quality of any given imaging experiment. If the SNR is not high enough, it becomes impossible to differentiate tissues from one another or the background.

The choice of imaging parameters such as the number of repetitions, the number of $k$-space samples $\left(N_{\mathrm{x}}, N_{\mathrm{y}}\right.$, and $\left.N_{\mathrm{z}}\right)$, the readout bandwidth, and voxel dimensions $(\Delta x, \Delta y$, and $\Delta z$ ), directly affect the SNR of the resulting data sets (see Equation B6.2.8); therefore, it is critical to know how SNR depends on imaging parameters prior to designing an MR protocol.

The SNR depends on many parameters. We first give its general form and then discuss the significance of imaging parameters:

$$
\mathrm{SNR} / \text { voxel } \propto \Delta x \Delta y \Delta z \sqrt{N_{\mathrm{acq}} N_{\mathrm{y}} N_{\mathrm{z}} T_{\mathrm{s}}}
$$

where $T_{\mathrm{s}}=N_{\mathrm{x}} \Delta t$ and $N_{\mathrm{acq}}$ is the number of acquisitions. Note that $\mathrm{SNR} /$ voxel in Equation B6.2.1 indicates the SNR per voxel. One commonly used approach for improving SNR is repeating the entire imaging experiment $N_{\text {acq }}$ times and averaging the signal over these $N_{\text {acq }}$ measurements. Although this simple approach improves SNR as the square root of the number of acquisitions, the total imaging time increases as the number of acquisitions. The SNR dependence on other imaging parameters is quite complicated, since these parameters are interrelated; therefore, prior to varying a specific imaging parameter to improve SNR, its effects on other imaging parameters need to be checked. For example, if $N_{\mathrm{y}}$ is doubled but $L_{\mathrm{y}}$ (field-of-view) is fixed, then $\Delta y$ is also cut in half and SNR drops by $\sqrt{2}$. Mathematically, it is possible to improve SNR by degrading resolution while increasing the total sampling time; however, there is a limit as to how long $T_{\mathrm{s}}$ can be before the signal completely dephases due to $T_{2}{ }^{*}$ relaxation. Several practical cases are given in Table B6.2.1 and Table B6.2.2 to illustrate the SNR dependence on read and phase encoding direction parameters, including spatial resolution, field-of-view, and sampling size. Generally, the larger the voxel size (the lower the resolution), the better the SNR will be (see Equation B6.2.1). This trade-off between resolution and SNR is illustrated with a phantom study as shown in Figure B6.2.1.

SNR also depends on inherent tissue parameters and field strength. For blood, the $T_{1}$ relaxation time can be reduced to improve SNR by the injection of a contrast agent. In addition, SNR can be improved with increased field strength; however, finding the optimal field strength for imaging has always been complicated and controversial. This is due to the fact that many factors may affect the outcome. Perhaps the most important are $T_{1}$ and the effects of field inhomogeneity, all of which lead to modifications of SNR as a function of field strength. In this unit, we will give a prediction of the signal as a function of field strength and a practical estimate of the ratio of the significance of the effective resistance and thermal noise at one field strength.

Contributed by Azim Celik and Weili Lin

Current Protocols in Magnetic Resonance Imaging (2002) B6.2.1-B6.2.9

Copyright $\odot 2002$ by John Wiley \& Sons, Inc.
Signal, Noise, and Contrast

B6.2.1

Supplement 4 


\section{TECHNICAL DISCUSSION}

\section{SNR Dependence on Imaging Parameters}

Signal and noise have been discussed separately in UNIT B6.1; however, signal or noise information by itself does not determine the quality of an image. It is the ratio of signal intensity to noise standard deviation (SNR) which determines image quality; therefore, it is important to be aware of the imaging parameter effects on SNR prior to designing a clinical or research protocol.

\section{Improving SNR by averaging over multiple acquisitions}

Repeating an entire imaging experiment $N_{\text {acq }}$ times and averaging the signal over these $N_{\text {acq }}$ measurements to improve the SNR is a common practice. The MRI system typically adds the signals directly to one another, and does not store them separately, saving a great deal of data space. The averaged $k$-space sample $s_{\mathrm{m}, \mathrm{av}}(k)$ of $s_{\mathrm{m}}(k)$ is:

$$
s_{\mathrm{m}, \mathrm{av}}(k)=\frac{1}{N_{\mathrm{acq}}} \sum_{\mathrm{i}=1}^{N_{\mathrm{acq}}} s_{\mathrm{m}, \mathrm{i}}(k)
$$

This implies that

$$
\overline{s_{\mathrm{m}, \mathrm{av}}(k)}=\frac{1}{N_{\mathrm{acq}}} \sum_{\mathrm{i}=1}^{N_{\mathrm{acq}}} \overline{s_{\mathrm{m}, \mathrm{i}}(k)}=\frac{1}{N_{\mathrm{acq}}}\left(N_{\mathrm{acq}} s(k)\right)=s(k)
$$

The noise from each of the $N_{\text {acq }}$ acquisitions is assumed to be statistically independent from one acquisition to the next. As a result, the noise variance $\sigma_{m}^{2}$ from each measurement adds in quadrature to the total noise variance of the averaged signal $s_{\mathrm{m}, \mathrm{av}}(k)$, i.e.:

$$
\sigma_{\mathrm{m}, \mathrm{av}}^{2} \equiv \operatorname{var}\left(s_{\mathrm{m}, \mathrm{av}}(k)\right)=\frac{1}{N_{\mathrm{acq}}^{2}} \sum_{\mathrm{i}=1}^{N_{\mathrm{acq}}} \operatorname{var}\left(s_{\mathrm{m}, \mathrm{i}}(k)\right)=\frac{\sigma_{\mathrm{m}}^{2}(k)}{N_{\mathrm{acq}}}
$$

Therefore, the standard deviation of the noise is given by

$$
\sigma_{\mathrm{m}, \mathrm{av}}(k)=\frac{\sigma_{\mathrm{m}}(k)}{\sqrt{N_{\mathrm{acq}}}}
$$

The SNR of the $k$-space signal becomes

$$
\operatorname{SNR}(k)=\frac{\overline{s_{\mathrm{m}, \mathrm{av}}(k)}}{\sigma_{\mathrm{m}, \mathrm{av}}(k)}=\sqrt{N_{\mathrm{acq}}} \frac{s(k)}{\sigma_{\mathrm{m}}(k)}
$$

i.e., the SNR improves as the square root of the number of acquisitions if the noise is uncorrelated from one experiment to the next; however, other sources of systematic noise from the MR experiment can lead to $\sigma_{m}^{2}$ being greater than $\sigma_{\text {thermal }}^{2}$ and these sources will not be reduced the same way by averaging.

Signal-to-Noise Ratio as a Function of Imaging Parameters

B6.2.2
The noise for a given voxel has already been shown to be proportional to $\sigma_{\mathrm{m}}$ (UNIT B6.1). Hence, the same $\sqrt{N_{\text {acq }}}$ dependence carries over into the expression for the SNR/voxel, i.e.: 


$$
\mathrm{SNR} / \operatorname{voxel}(p \Delta x, q \Delta y, r \Delta z) \propto \frac{\sqrt{N_{\mathrm{acq}}}}{\sigma_{0}}
$$

as well.

\section{Generalized dependence of SNR in a 3-D sequence on imaging parameters}

The SNR dependence on imaging parameters is complicated by the noise behavior. Noise depends on many different imaging parameters. From Equations B6.1.9, B6.1.10, B6.1.26, and B6.2.5:

$$
\mathrm{SNR} / \text { voxel } \propto \frac{\Delta x \Delta y \Delta z \sqrt{N_{\text {acq }}}}{\sqrt{\frac{\mathrm{BW} \text { read }}{N_{\mathrm{x}} N_{\mathrm{y}} N_{\mathrm{z}}}}}
$$

Substituting $\mathrm{BW}_{\text {read }}=1 / \Delta t$ (as given in Chapter B4) yields:

$$
\mathrm{SNR} / \text { voxel } \propto \Delta x \Delta y \Delta z \sqrt{N_{\mathrm{acq}} N_{\mathrm{x}} N_{\mathrm{y}} N_{\mathrm{z}} \Delta t}
$$

Since $T_{\mathrm{s}}=N_{\mathrm{x}} \Delta t$, substituting this into Equation B6.2.9 yields:

$$
\mathrm{SNR} / \text { voxel } \propto \Delta \mathrm{x} \Delta \mathrm{y} \Delta \mathrm{z} \sqrt{\mathrm{N}_{\mathrm{acq}} N_{\mathrm{y}} N_{\mathrm{z}} T_{\mathrm{s}}}
$$

Equations B6.2.8 to B6.2.10 can be rewritten in a number of ways, depending on the parameters. It is necessary to keep in mind that even though any parameters in Equation B6.2.8 to Equation B6.2.10 may be varied without altering the validity of the relations, the following relations, as derived in Chapter B4, hold:
(a) $\quad L_{\mathrm{x}}=N_{\mathrm{x}} \Delta x$
(b) $\quad L_{\mathrm{y}}=N_{\mathrm{y}} \Delta y$
(c) $L_{\mathrm{z}}=N_{\mathrm{z}} \Delta z$
(d) $T_{\mathrm{s}}=N_{\mathrm{x}} \Delta t$
(e) $\mathrm{BW}_{\text {read }}=\frac{1}{\Delta t}=\gamma G_{\mathrm{x}} L_{\mathrm{x}}$
(f) Bandwidth per voxel $=\mathrm{BW}_{\text {read }} / N_{\mathrm{x}}$

These interrelations exist implicitly in each of the above expressions for the SNR; therefore, whenever a parameter in a given expression for SNR is varied, the resultant effects on the rest of the parameters must be checked. Also, it is obvious that through these relations a number of other expressions for the SNR may be developed to highlight the effects of varying a certain subset of these parameters. Often an expression for SNR will be accompanied by a condition that some quantity be kept constant, which limits how certain parameters may be varied.

To calculate what happens in the case of a fixed variable, an implicit form can be substituted into Equation B6.2.10. For example, since $\Delta x=L_{\mathrm{x}} / N_{\mathrm{x}}$, if $\Delta x$ is fixed, $L_{\mathrm{x}}$ and $N_{\mathrm{x}}$ cannot vary arbitrarily and Equation B6.2.9 or Equation B6.2.10 is better written as: 


$$
\mathrm{SNR} / \text { voxel }\left.\propto L_{\mathrm{x}} \Delta y \Delta z \sqrt{\frac{N_{\mathrm{y}} N_{\mathrm{z}} \Delta t}{N_{\mathrm{x}}}}\right|_{\Delta x=L_{\mathrm{x}} / N_{\mathrm{x}}=\text { constant }}
$$

Other such specialized proportionalities can be derived.

An increase in the spatial resolution by a factor of two in both in-plane directions with fixed fields of view leads to a factor of two loss in SNR when the increased spatial

A

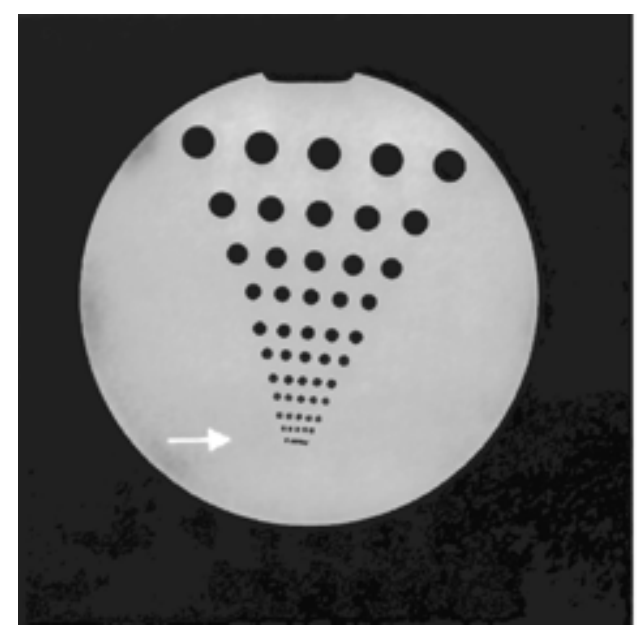

C

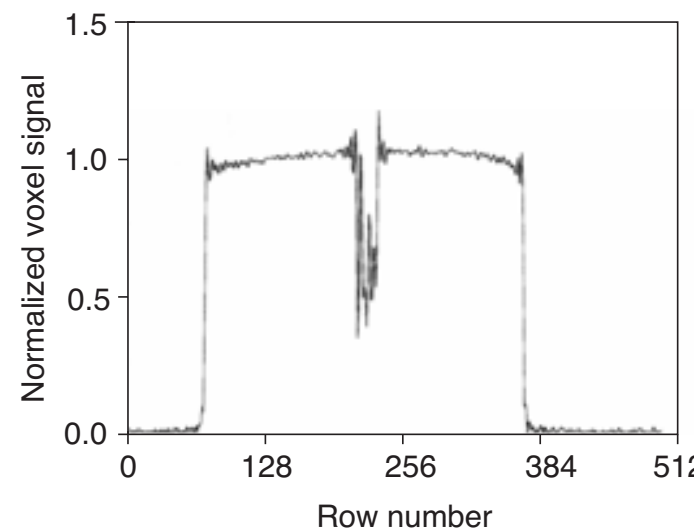

$\mathrm{B}$

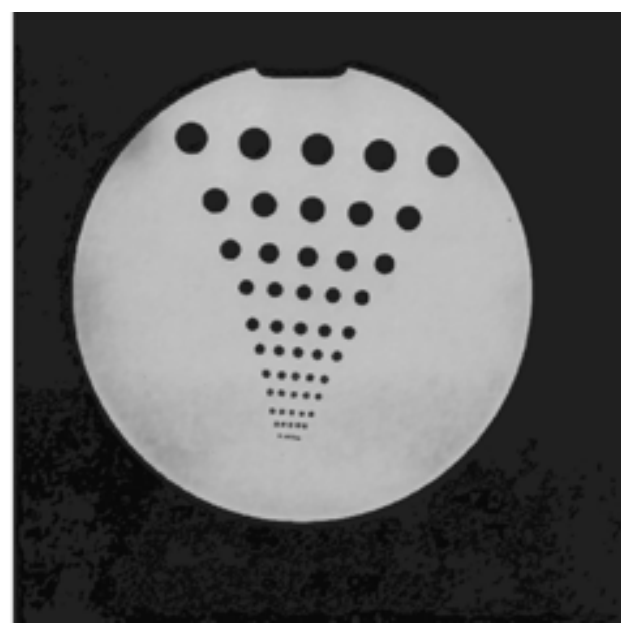

$\mathrm{D}$

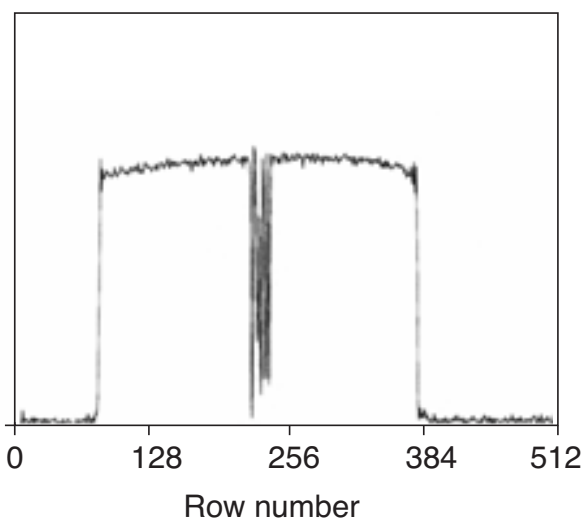

Figure B6.2.1 Two images collected with identical $T_{\mathrm{R}}, T_{\mathrm{E}}$, and $G_{\mathrm{x}}$; however, $N_{\mathrm{x}}, N_{\mathrm{y}}$, and $T_{\mathrm{S}}$ in (B) are two times of the same as those in (A), leading to an improvement in spatial resolution. This increase in resolution leads to a reduction of the SNR by a factor of 2 . Noise standard deviation inside the object is estimated by taking $1 / 1.253 \cong 0.8$ times the mean measured in a region outside of the phantom where there is no signal. This noise-only region has to lie reasonably away from the edge of the object, and there should be no artifacts nearby. The profiles in (D) and (C) illustrate the larger variation in noise for (B) versus (A), respectively. They also show the higher resolution associated with (D), where five distinct dips (each corresponding to one resolution element) are visible versus only three in (C). The profiles were taken through the row cutting through the last row of the smallest resolution elements. The imaging parameters used were (A) $T_{\mathrm{R}} / T_{\mathrm{E}}=$ $1000 \mathrm{msec} / 14 \mathrm{msec}, \theta=90^{\circ}, T_{\mathrm{s}}=11.2 \mathrm{msec}, N_{\text {acq }}=1, \Delta x \times \Delta y \times \Delta z=0.5 \mathrm{~mm} \times 0.5 \mathrm{~mm} \times 2.0 \mathrm{~mm}, N_{\mathrm{x}} \times N_{\mathrm{y}}$ $=512 \times 384, \tau_{\mathrm{RF}}=2.56 \mathrm{msec}, \mathrm{G}_{\mathrm{ss}}=6 \mathrm{mT} / \mathrm{m}$; (B) $T_{\mathrm{s}}=5.6 \mathrm{msec}, \Delta x \times \Delta \mathrm{y} \times \Delta z=1.0 \mathrm{~mm} \times 1.0 \mathrm{~mm} \times 2.0 \mathrm{~mm}$, $N_{\mathrm{x}} \times N_{\mathrm{y}}=256 \times 192$. 
resolution is achieved by maintaining the read gradient fixed while $T_{\mathrm{s}}\left(\right.$ or $\left.N_{\mathrm{x}}\right)$ is doubled (from Equation B6.2.10). Such an example is shown in Figure B6.2.1, from which the mean and standard deviation estimates were obtained. Taking the ratio of the mean of the background to the noise standard deviation in the two cases demonstrates the consistency of the measured SNR with that expected from Equation B6.2.10. Doing this in a region where the profile is flat yields an SNR/voxel of 187.5 for Figure B6.2.1A and 91.0 for Figure B6.2.1B.

\section{SNR Dependence on Read Direction Parameters}

From Equation B6.2.10, with all other parameters maintained constant, the SNR dependence on read direction parameters can be reduced to

$$
\mathrm{SNR} / \text { voxel }\left.\right|_{\text {read }} \propto \Delta x \sqrt{T_{\mathrm{s}}}
$$

Although Equation B6.2.13 depends only upon two parameters, dependencies on $L_{\mathrm{x}}$, $\mathrm{BW}_{\text {read }}$, etc. are implicit in this expression (see Equations B6.2.10 and B6.2.11). Due to the importance of understanding the effects of altering read direction parameters, several example situations are shown in Table B6.2.1. Case 1 is chosen as a reference and given an SNR of unity. Also, note that in order to simplify the treatment, all of the situations in Table B6.2.1 are restricted to doubling or halving the parameters involved. There are also practical aspects to these choices, which will be discussed below.

From Equation B6.2.13, it appears that it might be possible to obtain very high resolution without reducing SNR by shrinking $\Delta x$ while increasing $T_{\mathrm{s}}$. Realistically, however, there is a limit as to how long $T_{\mathrm{s}}$ can be before the signal is seriously degraded by $T_{2}{ }^{*}$ effects.

\section{Oversampling to avoid aliasing}

Although the topic of aliasing has been dealt with in Chapter B4, it is useful here to revisit it in terms of how changing the FOV (field of view) affects the SNR in a given experiment. In Table B6.2.1, cases 2 and 3 demonstrate that altering the FOV does not alter the SNR

Table B6.2.1 SNR/Voxel: When Voxel Size Is Changed in the Read Direction Under Different Conditions $^{a}$

\begin{tabular}{|c|c|c|c|c|c|c|c|}
\hline Case no. & $\Delta x$ & $N_{\mathrm{x}}$ & $L_{\mathrm{x}}$ & $G_{\mathrm{x}}$ & $\Delta t$ & $T_{\mathrm{s}}$ & SNR \\
\hline \multicolumn{8}{|c|}{ Reference case } \\
\hline 1 & $\Delta x_{0}$ & $N_{0}$ & $L_{0}$ & $G_{0}$ & $\Delta t_{0}$ & $T_{\mathrm{s}, 0}$ & 1 \\
\hline \multicolumn{8}{|c|}{ Data reduction and oversampling } \\
\hline 2 & $\Delta x_{0}$ & $N_{0} / 2$ & $L_{0} / 2$ & $G_{0}$ & $2 \Delta t_{0}$ & $T_{\mathrm{s}, 0}$ & 1 \\
\hline 3 & $\Delta x_{0}$ & $2 N_{0}$ & $2 L_{0}$ & $G_{0}$ & $\Delta t_{0} / 2$ & $T_{\mathrm{s}, 0}$ & 1 \\
\hline \multicolumn{8}{|c|}{ Degrading spatial resolution } \\
\hline 4 & $2 \Delta x_{0}$ & $N_{0} / 2$ & $L_{0}$ & $G_{0}$ & $\Delta t_{0}$ & $T_{\mathrm{s}, 0} / 2$ & $\sqrt{2}$ \\
\hline 5 & $2 \Delta x_{0}$ & $N_{0} / 2$ & $L_{0}$ & $G_{0} / 2$ & $2 \Delta t_{0}$ & $T_{\mathrm{s}, 0}$ & 2 \\
\hline \multicolumn{8}{|c|}{ Improving spatial resolution } \\
\hline 6 & $\Delta x_{0} / 2$ & $N_{0}$ & $L_{0} / 2$ & $G_{0}$ & $2 \Delta t_{0}$ & $2 T_{\mathrm{s}, 0}$ & $1 / \sqrt{2}$ \\
\hline 7 & $\Delta x_{0} / 2$ & $N_{0}$ & $L_{0} / 2$ & $2 G_{0}$ & $\Delta t_{0}$ & $T_{\mathrm{s}, 0}$ & $1 / 2$ \\
\hline 8 & $\Delta x_{0} / 2$ & $2 N_{0}$ & $L_{0}$ & $2 G_{0}$ & $\Delta t_{0} / 2$ & $T_{\mathrm{s}, 0}$ & $1 / 2$ \\
\hline 9 & $\Delta x_{0} / 2$ & $2 N_{0}$ & $L_{0}$ & $G_{0}$ & $\Delta t_{0}$ & $2 T_{\mathrm{s}, 0}$ & $1 / \sqrt{2}$ \\
\hline
\end{tabular}

${ }^{a}$ The SNR/voxel is given with respect to that of case 1 and can be seen to correlate exactly with $\Delta x\left(T_{\mathrm{S}}\right)^{1 / 2}$ or equivalently, $\left(\Delta x / G_{\mathrm{X}}\right)^{1 / 2}$.

Signal, Noise, and Contrast

B6.2.5

Supplement 4 
of an experiment as long as $T_{\mathrm{s}}$ and $\Delta x$ are unchanged. There are two important implications of this result.

First, it is possible to avoid aliasing in a given experiment by doubling the FOV in the read direction. This is accomplished by collecting twice as many points without varying the read gradient $G_{\mathrm{x}}$ or $T_{\mathrm{s}}$ (referred to as oversampling; case 3), which neither degrades nor improves the SNR of the experiment. Therefore, in many practical imaging situations, oversampling is used to double the FOV in the read direction and reduce aliasing artifacts without sacrificing $S N R$, lengthening $T_{s}$, or the acquisition time.

Consider acquiring a transverse image of the human chest-e.g., where the left to right dimension of the chest is very large and is most likely to be chosen as the read direction. Oversampling in the read direction is then used to guarantee that the FOV extends past the patient's arms without increasing imaging time, or degrading SNR. The data are usually oversampled by a factor of two and then just the central data are kept. In this way, the arms don't even appear in the image because the outer quarters of the originally reconstructed image are discarded. Higher levels of oversampling are also possible. This may prove useful for sagittal or coronal imaging when a large coil or surface coil is used and the region of interest is small. Alternatively, for a small object, where aliasing is not a problem and data storage space is a premium, choosing a smaller FOV and collecting fewer data points (case 2) does not reduce SNR.

\section{Degrading resolution to increase $S N R$}

In case $4, \Delta x$ is increased by a factor of 2 , the number of data points collected is halved, and $T_{\mathrm{s}}$ is also halved. As seen from Table B6.2.1, SNR is increased by $\sqrt{2}$. If lower resolution can be tolerated, this increase in SNR could lead to a better tissue recognition, and the reduction in $T_{\mathrm{s}}$ could be beneficial in reducing the chemical shift artifact, in overcoming static field inhomogeneity effects (since bandwidth per voxel is increased), and in reducing relaxation effects during sampling.

In case 5, resolution is reduced by a factor of 2 and SNR is doubled because this increase in resolution is achieved while $T_{\mathrm{s}}$ is held constant. A doubling of SNR can significantly improve signal quality. Note that this effect is achieved by reducing the read gradient by a factor of 2 . In most cases, reducing the gradient is not a problem, but it must be kept in mind that if the applied gradient strength reduces to a level comparable to those produced by local field inhomogeneities, then severe image distortion will occur. This trick of using a fixed $T_{s}$ with the read gradient halved to acquire low resolution images and obtain a factor of 2 improvement in SNR is commonly used in clinical applications.

\section{Improving resolution in the read direction}

In both cases 6 and 7, $\Delta x$ is halved by reducing the FOV by a factor of 2 without changing the number of sampled points. This should be done only if the FOV in case 1 is greater than or equal to twice the width of the object in the read direction so that aliasing will be avoided. In case $6, T_{\mathrm{s}}$ is doubled by doubling $\Delta t$. This method leads to a reduction of SNR of only $\sqrt{2}$ since bandwidth per voxel is again halved. Realistically, $T_{\mathrm{s}}$ must be short enough in case 1 so that when it is doubled, it is still much shorter than $T_{2}^{*}$. In case $7, L_{\mathrm{x}}$ is halved by doubling the read gradient $G_{\mathrm{x}}$. This approach requires that enough gradient power be available to double $G_{\mathrm{x}}$. Unfortunately, SNR is reduced by a factor of 2, making this a very inefficient approach.

Signal-to-Noise

Ratio as a

Function of Imaging

Parameters

B6.2.6
In case $8, \Delta x$ is halved by doubling $N_{\mathrm{x}}$ and keeping $L_{\mathrm{x}}$ and $T_{\mathrm{s}}$ fixed, which requires doubling $G_{\mathrm{x}}$ and halving $\Delta t_{0}$. This also leads to a halving of the SNR. In case $9, \Delta x$ is halved by doubling $N_{\mathrm{x}}$ while keeping both $L_{\mathrm{x}}$ and $G_{\mathrm{x}}$ fixed. This requires doubling the total sampling time, but is accompanied by only a $\sqrt{2}$ reduction in SNR. This is probably the optimal 
way to double the resolution of the experiment as long as the increased $T_{\mathrm{s}}$ is not comparable to $T_{2}{ }^{*}$, and storing the extra data is not a problem, although it is similar to case 6 which requires less storage space but a smaller object.

Since $T_{\mathrm{s}}$ equals $1 /\left(₹ G_{\mathrm{x}} \Delta x\right)$, the proportionality in Equation B6.2.13 can be rewritten as

$$
\mathrm{SNR} / \text { voxel } \propto \sqrt{\frac{\Delta x}{G_{\mathrm{x}}}}
$$

A better understanding and feel for the SNR/voxel variation in the read direction can be obtained by relating the $\mathrm{SNR} /$ voxel to a combination of $\Delta x$ and bandwidth per voxel. To this end, Equation B6.2.14 can be rewritten in terms of bandwidth per voxel as follows:

$$
\mathrm{SNR} / \text { voxel } \propto \Delta x \sqrt{T_{\mathrm{s}}}=\Delta x / \sqrt{\mathrm{BW}_{\text {read }} / N_{\mathrm{x}}}=\Delta x / \sqrt{\text { bandwith per voxel }}
$$

where we have used $T_{\mathrm{s}}=N_{\mathrm{x}} \Delta t=N_{\mathrm{x}} / \mathrm{BW}_{\text {read }}$.

Both Equations B6.2.14 and B6.2.15 contain no hidden dependencies and require none of the imaging parameters to be fixed-i.e., they can be applied to any situation for computing the relative SNR change. According to these expressions, the SNR/voxel decreases only by a factor of $\sqrt{2}$ for every halving of $\Delta x$ as long as the read gradient is fixed. SNR, however, reduces by a factor of 2 if this improvement in spatial resolution is accompanied by a doubling of $G_{\mathrm{x}}$. Note the consistency with these observations for cases 6 through 9 in Table B6.2.1 and in Figure B6.2.2.

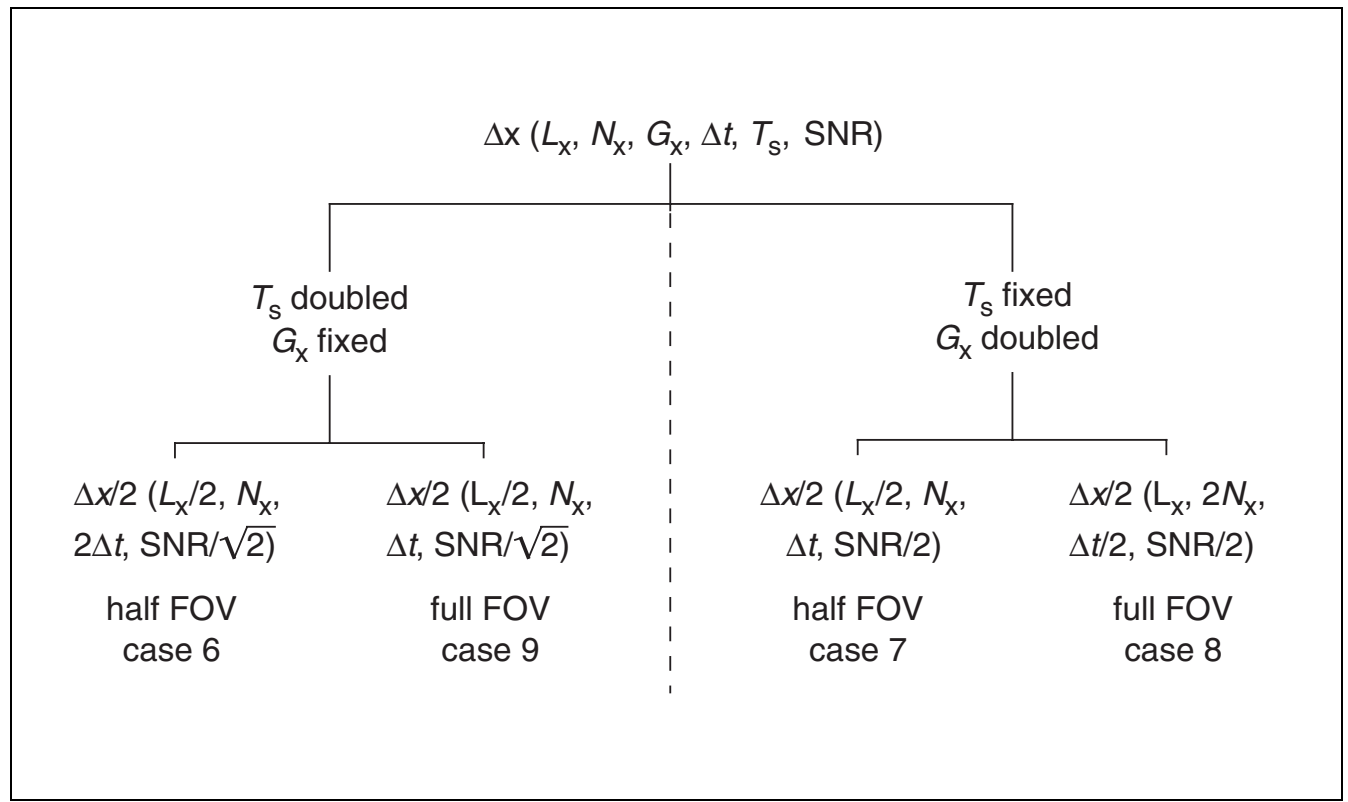

Figure B6.2.2 Different ways of achieving improved spatial resolution in the read direction, and their effects on SNR. This figure summarizes cases 6 through 9 in Table B6.2.1, showing also what parameters were changed in comparison with case 1 to attain high resolution. For a fixed $T_{\mathrm{S}}$, either approach yields a loss of 2 in SNR while for $T_{\mathrm{s}}$ doubled, only a loss of $\sqrt{2}$ in SNR occurs.

Signal, Noise, and Contrast

B6.2.7 


\section{SNR Dependence on Phase Encoding Parameters}

Pulling out just the SNR dependence on parameters which change only the image characteristics in the in-plane and through-plane phase encoding directions $(\hat{y}$ and $\hat{z}$, respectively) gives

$$
\mathrm{SNR} / \text { voxel } \propto \Delta y \Delta z \sqrt{N_{\mathrm{y}} N_{\mathrm{z}}}
$$

(recall $L_{\mathrm{y}}=N_{\mathrm{y}} \Delta y$ and $L_{\mathrm{z}}=N_{\mathrm{z}} \Delta z$ ). There are only two alternate methods for improving spatial resolution in the phase encoding directions: first, decreasing $\Delta y$ or $\Delta z$ by increasing $N_{\mathrm{y}}$ or $N_{\mathrm{z}}$ while keeping $L_{\mathrm{y}}$ or $L_{\mathrm{z}}$ fixed (case 3 in Table B6.2.2), or second, decreasing $\Delta y$ or $\Delta z$ by decreasing $L_{\mathrm{y}}$ or $L_{\mathrm{z}}$ while keeping $N_{\mathrm{y}}$ or $N_{\mathrm{z}}$ fixed (case 2 in Table B6.2.2).

Consider halving $\Delta y$ using either of these methods. In the first method, the SNR decreases only by a factor of $\sqrt{2}$, whereas in the second method, the SNR decreases by a factor of 2. What are the advantages or disadvantages of either method? The first method, requiring doubling $N_{\mathrm{y}}$, takes twice as long to complete compared to that of the second method. If the SNR is good enough and aliasing is avoided, the second method is twice as fast as the first while having $\sqrt{2}$ worse SNR in comparison with the first approach. Of course, in the same imaging time as required by the first method, two acquisitions can be performed with the second method to reclaim the factor of $\sqrt{2}$ SNR loss, while maintaining the advantage of requiring less image storage space. These two cases are highlighted in Table B6.2.2.

Table B6.2.2 Two Different Ways to Improve Spatial Resolution in the Phase Encoding Direction, and Their Effects on the SNR and Total Imaging Time

\begin{tabular}{lcccccc}
\hline Case no. & $\Delta y$ & $N_{\mathrm{y}}$ & $L_{\mathrm{y}}$ & $G_{\mathrm{y}, \max }$ & $T_{\mathrm{T}}$ & SNR \\
\hline \multicolumn{2}{l}{ Reference case } \\
1 & \multicolumn{1}{c}{$\Delta y_{0}$} & $N_{\mathrm{y}, 0}$ & $L_{\mathrm{y}, 0}$ & $G_{\mathrm{y}, 0}$ & $T_{\mathrm{T}_{0}}$ & 1 \\
\multicolumn{2}{l}{ Improved spatial resolution } \\
2 & $\Delta y_{0} / 2$ & $N_{\mathrm{y}, 0}$ & $L_{\mathrm{y}, 0} / 2$ & $2 G_{\mathrm{y}, 0}$ & $T_{\mathrm{T}_{0}}$ & $1 / 2$ \\
3 & $\Delta_{\mathrm{y} 0} / 2$ & $2 N_{\mathrm{y}, 0}$ & $L_{\mathrm{y}, 0}$ & $2 G_{\mathrm{y}, 0}$ & $2 T_{\mathrm{T}_{0}}$ & $1 / \sqrt{2}$ \\
\hline
\end{tabular}

\section{SNR in 2-D Imaging}

The SNR expression in Equation B6.2.10 can be rewritten for a 2-D imaging experiment. The voxel now has dimensions of $\Delta x \times \Delta y \times \mathrm{TH}$, where $\mathrm{TH}$ is the slice thickness. Also, $N_{\mathrm{z}}$ is replaced by unity; therefore:

$$
\left.(\mathrm{SNR} / \text { voxel })\right|_{2-D} \propto \Delta x \Delta y \mathrm{TH} \sqrt{N_{\mathrm{y}} T_{\mathrm{s}}}
$$

Signal-to-Noise

Ratio as a Function of Imaging

Parameters

B6.2.8

In other words, a 2-D imaging experiment performed with exactly the same imaging parameters (including $T_{\mathrm{R}}, T_{\mathrm{E}}$ and flip angle) with $\mathrm{TH}=\Delta z$ has $\sqrt{N_{\mathrm{z}}}$ worse SNR in comparison with the 3-D imaging experiment; however, the 2-D imaging experiment requires an imaging time which is $N_{\mathrm{z}}$ times shorter than the 3-D experiment. It is typically possible to collect the data for only one slice per $T_{\mathrm{R}}$ when the $T_{\mathrm{R}}$ value in the 2-D imaging experiment equals the practically sensible choice of short $T_{\mathrm{R}}$ in the 3-D imaging experiment. To obtain the same spatial coverage in the slice select direction requires $N_{\mathrm{z}}$ imaging experiments, increasing the total imaging time by $N_{\mathrm{z}}$ in the 2-D imaging case. If the same imaging time is used for both the 2-D and 3-D imaging experiments, the same volume of coverage and the same contrast as the 3-D can be achieved in 2-D imaging albeit only 
with $\sqrt{N_{\mathrm{z}}}$ less SNR. Alternatively, a single slice with the same SNR as the 3-D experiment (obtained by imaging with $N_{\text {acq2-D }}=N_{\mathrm{z}}$ ) can be obtained. The most efficient means of collecting 2-D data and covering the same region-of-interest as in the 3-D imaging case is to use $T_{\mathrm{R}_{2-\mathrm{D}}}=N_{\mathrm{z}} \mathrm{T}_{\mathrm{R}_{3-\mathrm{D}}}$ and use a multi-slice acquisition (although this guarantees neither that the 2-D SNR is as good as the 3-D SNR, as discussed above, nor that the contrast is comparable with the 3-D imaging method).

\section{KEY REFERENCES}

Escanyé, J.M., Canet, D., and Robert, J. 1982. Frequency dependence of water proton longitudinal nuclear magnetic relaxation times in mouse tissues at $20^{\circ} \mathrm{C}$. Biochim. Biophys. Acta 721:305.

The square root field strength dependence of $T_{1}$ was proposed in this article.

Haacke, E.M., Brown, R.W., Thompson, M.R., and Venkatesan, R. 1999. Magnetic Resonance Imaging: Physical Principles and Sequence Design. John Wiley \& Sons, New York.

The SNR as a function of imaging parameters and field strength is described in detail in this text.

Contributed by Azim Celik

General Electric Company

Milwaukee, Wisconsin

Weili Lin

The University of North Carolina at Chapel Hill

Chapel Hill, North Carolina 